\title{
ON SOME PROPERTIES OF THE SPACES OF ALMOST CONTINUOUS FUNCTIONS*
}

\author{
RYSZARD JERZY PAWLAK† \\ Institute of Mathematics \\ Lódź University \\ Banacha 22, 90-238 Lódź, POLAND
}

(Received May 1, 1992 and in revised form October 31, 1994)

\begin{abstract}
In this paper we prove that, in the space $\mathcal{A}$ of almost continuous functions (with the metric of uniform convergence), the set of functions of the first class of Baire is superporous at each point of this space
\end{abstract}

KEY WORDS AND PHRASES: Almost contınuity; the first class of Baire. blockıng set. superporosity 1991 AMS SUBJECT CLASSIFICATION CODES: 54C35; 54C08. 26A15. 26A21

Among many generalizations of the notion of continuity, special attention of mathematicians has recently been focused on the concept of almost continuous (in the sense of J. Stallings [8]) functions. The observation of the fact that these functions, in the case of the mapping of closed intervals into themselves, possess a fixed point caused the investigation of topological properties of those mappings (e.g. [1], [3], [5]). On the other hand, investigations connected with algebraic operations performed on almost continuous functions were carried out (e.g. [4], [9], [6]). However, so far, less attention has been devoted to the study of the structure of the space of almost continuous functions with the topology of uniform convergence (and other topologies defined in this set of functions).

The present paper is an attempt at a preparatory study of the basic properties of this space.

On the basis of J.B. Brown's results included in paper [1], we may infer that every Darboux function of the first class of Baire is an almost continuous function. Of course, every almost continuous function is a Darboux function, but need not be a function of the first class of Baire $([1],[7])$. Consequently, the following question arises: "how large" is the set in the space of almost continuous functions $f: I \rightarrow I$ with the metric of uniform confergence, composed of functions of the first class of Baire? The answer to this question is contained in Theorem 1 . It should be stressed here that this theorem thereby answers the question how often functions of the first class of Baire occur in the class of Darboux functions $f: I \rightarrow I$ possessing a fixed point.

Before we formulate the theorem and prove the lemmas preceding this theorem, we give the essential definitions and symbols used in this paper.

Throughout the work, we shall consider only functions $f: I \rightarrow I$, where $I=[0,1]$. Let $I^{2}=I \times I$. The symbol $\varrho$ stands for the natural metric in $I^{2}$.

The bilateral closed (bilateral open, etc.) segment with the end-points $a$ and $b$ is denoted by $[a, b]((a, b)$, etc. $)$. Since our cosiderations are restricted to the unit interval, we write $[a, b]$ instead $[a, b] \cap I$ (similarly $(a, b)$ instead of $(a, b) \cap I$, etc.).

The graph of the function $f$ is denoted by $\mathcal{G}(f)$. 
We say that a function $f: X \rightarrow Y$, where $X$ and $Y$ are arbitrary topological spaces, is almost continuous if, for any open set $V \subset X \times Y$ containing the graph $\mathcal{G}(f), V$ contains the graph of some continuous function $g: X \rightarrow Y$.

The set of all almost continuous functions $f: I \rightarrow I$ and the metric space consisting of these functions with the metric $\varrho^{*}$ of uniform convergence is denoted by the symbol $\mathcal{A}$. By the letter $\mathcal{A}_{1}$ we denote the set of all $f \in \mathcal{A}$ which are functions of the first class of Baire.

Suppose $f: X \rightarrow Y$. The statement that the subset $\dot{C}$ of $X \times Y$ is a blocking set of $f$ in $X \times Y$ means that $C$ is closed relative to $X \times Y, C \cap \mathcal{G}(f)=\emptyset$ and $C$ intarsects $\mathcal{G}(g)$ whenever $g: X \rightarrow Y$ is a continuous function. If no proper subset of $C$ is a blocking set of $f$ in $X \times Y, C$ is said to be the minimal blocking set of $f$ in $X \times Y$.

Let $A \subset I^{2}$. Then $\operatorname{proj}_{X}(A)$ denotes the projection of the set $A$ onto the $X$-axis and, in the case when $A$ is a closed set, we assume $(A)_{\min }=\left\{\left(x_{0}, y_{0}\right) \in A: y_{0}=\min \left\{y:\left(x_{0}, y\right) \in A\right\}\right\}$.

Let $f: I \rightarrow I$. If $A \subset I(f(I) \subset B)$, then by the symbol $f_{\mid A}\left(f_{\| B}\right)$ we understand a function from $A$ to $I$ (from $I$ to $B$ as a subspace of $I$ ).

For $0 \leq \alpha<\beta \leq 1$, we define a function $f_{\beta}^{\alpha}: I \rightarrow I$ by letting:

$$
f_{\beta}^{\alpha}(x)= \begin{cases}f(x) & \text { if } f(x) \in[\alpha, \beta], \\ \alpha & \text { if } f(x) \leq \alpha, \\ \beta & \text { if } f(x) \geq \beta\end{cases}
$$

Let $X$ be a metric space. The open ball with centre $x$ and radius $r>0$ will be denoted by $K(x, r)$. Let $M \subset X, x \in X$ and $R>0$. Then we denote by $\gamma(x, R, M)$ the supremum of the set of all $r>0$ for which there exists $z \in X$ such that $K(z, r) \subset K(x, R) \backslash M$. The number $p(M, x)=2 \cdot \lim \sup _{R \rightarrow 0+} \frac{\gamma(x, R, M)}{R}$ is called the porosity of $M$ at $x([10]) . M$ is porous at $x$ if $p(M, x)>0$. We say that $E \subset X$ is superporous at $x \in X$ if $E \cup F$ is porous at $x$ whenever $E$ is porous at $x([11])$.

Now we formulate three lemmas showing some properties of almost continuous functions. These properties are applied later.

Lemma 1 Let $f: I \rightarrow I$ ba a function such that $f(I) \subset[\alpha, \beta]$ where $\alpha<\beta$. Then $f \in \mathcal{A}$ if and only if $f_{\|[\alpha, \beta]}$ is almost continuous.

Remark 1 If $f: X \rightarrow Y$, where $X, Y$ are toplogical spaces, is a continuous function such that $f(X) \subset B \subset Y$, then $f_{\| B}$ is a continuous function, too. However it is not difficult to construct an example of a topology $\mathcal{T}$ defined in $I$ and an almost continuous function $g: I \rightarrow(I, \mathcal{T})$ such that $g(I)=\left[\frac{1}{2}, 1\right]$ and $g_{\|\left[\frac{1}{2}, 1\right]}$ is not almost continuous.

Lemma 2 ([6]) A function $f: I \rightarrow I$ is almost continuous if and only if $f_{\|[a, b]}$ is almost continuous for any $a, b \in[0,1]$.

Remark 2 It is known that there exists an almost continuous real function defined on some compact subset $K$ of the plane, such that the restriction of this function to the set Int $K$ is not almost continuous ([6]).

Lemma 3 Let $f: I \rightarrow[\alpha, \beta]$ be a function for which there exists an interval $(a, b) \subset I$ such that $f_{\mid I \backslash(a, 1]}$ and $f_{\mid I \backslash[0, b)}$ are almost continuous and $f$ is not almost continuous. Then $\operatorname{proj}_{X}\left(K_{f}\right) \cap(a, b)$ is a nondegenerate interval, where $K_{f} \subset I \times[\alpha, \beta]$ is the minimal blocking set of $f$. 
Proof. It is sufficient to prove (see, for example, [3], Theorem 1; [9], Lemma 4; [6], Theorem 1.1.2) that $\operatorname{proj}_{X}\left(K_{f}\right) \cap(a, b) \neq \emptyset$. Assume, to the contrary, that $\operatorname{proj}_{X}\left(K_{f}\right) \cap(a, b)=\emptyset$. Thus, according to the above-cited theorems, either $K_{f} \subset\{(x, y): x \leq a\}$ or $K_{f} \subset\{(x, y): x \geq b\}$. Without loss of generality assume that $K_{f} \subset\{(x, y): x \leq a\}$. Of corse, $K_{f} \cap \mathcal{G}(f)=\emptyset$, and so, $K_{f} \cap \mathcal{G}\left(f^{*}\right)=\emptyset$ where $f^{*}=f_{\mid \backslash \backslash(a, 1]}$. Let us consider the set $A=((I \backslash(a, 1]) \times[a, \beta]) \backslash K_{f}$. Thus $A$ is a neighbourhood of $\mathcal{G}\left(f^{*}\right)($ in $(I \backslash(a, 1]) \times[\alpha, \beta])$. This means that $A$ contains the graph of some continuous function $g^{*}: I \backslash(a, 1] \rightarrow[\alpha, \beta]$. Let (for $x \in I$ )

$$
g(x)=\left\{\begin{array}{lll}
g^{*}(x) & \text { if } & x \in I \backslash(a, 1] \\
g^{*}(a) & \text { if } & x \geq a
\end{array}\right.
$$

Then $g$ is continuous and $K_{f} \cap \mathcal{G}(g)=\emptyset$, which is impossible because $K_{f}$ is the blocking set of $f$. The contradictions obtained ends the proof of the lemma.

Theorem 1 The set $\mathcal{A}_{1}$ is perfect and superporous at each point t of the space $\mathcal{A}$.

Proof. According to J.B. Brown's theorem and the well-known fact (see, for example, [2], Theorem 2.3.4]) that the limit of a uniformly convergent sequence of Darboux functions of the first class of Baire is a Darboux function of the first class of Baire, we deduce that $\mathcal{A}_{1}$ is closed in $\mathcal{A}$.

We shall show that $\mathcal{A}_{1}$ is dense in itself.

Let $f \in \mathcal{A}_{1}, \mu>0$ and let $x_{0} \in(0,1)$ be a continuity point of $f$. Then either $f\left(x_{0}\right)<1$ or $f\left(x_{0}\right)>0$. Without loss of generality assume that $f\left(x_{0}\right)<1$. Let now $0<\mu_{1}<\frac{\mu}{3}$ be a number such that $f\left(x_{0}\right)+2 \cdot \mu_{1}<1$. Then there exists $\delta>0$ such that $\left[x_{0}-\delta, x_{0}+\delta\right] \subset(0,1)$ and

$$
f\left(\left[x_{0}-\delta, x_{0}+\delta\right]\right) \subset\left(f\left(x_{0}\right)-\mu_{1}, f\left(x_{0}\right)+\mu_{1}\right) .
$$

Let us consider $g: I \rightarrow I$ defined by letting: $g(x)=0$ for $x \notin\left[x_{0}-\delta, x_{0}+\delta\right], g\left(x_{0}\right)=\mu_{1}$ and $g$ is linear in $\left[x_{0}-\delta, x_{0}\right]$ and $\left[x_{0}, x_{0}+\delta\right]$. Thus $g$ is continuous, and so (see [2], Theorem 2.3.2), $h=f+g \in \mathcal{A}_{1}$. It is not difficult to see that $f \neq h$ and $\varrho^{*}(f, h)<\mu$.

In this way the fact that $\mathcal{A}_{1}$ is perfect has been demonstrated.

Now, we shall show that $\mathcal{A}_{1}$ is superporous at each point $t$ of the space $\mathcal{A}$.

So, let $M$ be an arbitrary porous set at $t$. Assume the notation from the definition of a porous set. Let $p(M, t)=2 \alpha>0$. This means that there exists a sequence $\left\{R_{n}\right\}$ such that $R_{n} \searrow 0$ and

$$
\lim _{n \rightarrow \infty} \frac{\gamma\left(t, R_{n}, M\right)}{R_{n}}=\alpha
$$

Let $n$ be a fixed number. From the definition of $\gamma\left(t, R_{n}, M\right)$ it follows that (for $n$ sufficiently large) there are $d \in \mathcal{A}$ and $\psi_{n} \geq \gamma\left(t, R_{n}, M\right)-\frac{1}{2^{n}} \cdot R_{n}>0$, such that $K\left(d, \psi_{n}\right) \subset K\left(t, R_{n}\right) \backslash M$. Moreover,

$$
\text { if } \overline{K\left(d, \frac{\psi_{n}}{2}\right)} \cap \mathcal{A}_{1}=\emptyset \text {, then } \gamma\left(t, R_{n}, M \cup \mathcal{A}_{1}\right) \geq \frac{\psi_{n}}{2} \text {. }
$$

Let us consider the case when there exists $\varphi \in \overline{K\left(d, \frac{\psi_{n}}{2}\right)} \cap \mathcal{A}_{1}$. We infer that

$$
K\left(\varphi, \frac{\psi_{n}}{2}\right) \subset K\left(d, \frac{\psi_{n}}{2}\right) \subset K\left(t, R_{n}\right) .
$$

To simplify the notation, assume $\varepsilon=\frac{\psi_{n}}{2}$. Let $x_{0}$ be a continuity point of $\varphi$. Then there exists a nondegenerate interval $[a, b]$ such that $\varphi([a, b]) \subset\left(\varphi\left(x_{0}\right)-\frac{\varepsilon}{4}, \varphi\left(x_{0}\right)+\frac{\varepsilon}{4}\right)$. On the set of points 
belonging to $(a, b)$ we define the following equivalence relation:

$$
x * y \text { if and only if } x-y \in \mathcal{Q}
$$

where $\mathcal{Q}$ denotes the set of alf rational numbers.

Let $\mathcal{B}$ denote the set of all equivalence classes of the above relation. For each $x \in(a, b)$, let $B_{x} \in \mathcal{B}$ be a set such that $x \in B_{x}$. By the symbol $\xi$ we denote an arbitrary mapping from $\mathcal{B}$ onto $\mathbf{B}_{1}$ where $\mathbf{B}_{1}$ stands for the set of all functions of the first class of Baire from $I$ to $\left[\varphi\left(x_{0}\right)-\frac{\varepsilon}{4}, \varphi\left(x_{0}\right)+\frac{\varepsilon}{4}\right]$. Moreover, we define the function $g$ by letting

$$
g(x)=\left\{\begin{array}{lll}
\varphi(x) & \text { if } x \leq a \text { or } x \geq b \\
\left(\xi\left(B_{x}\right)\right)(x) & \text { if } x \in(a, b)
\end{array}\right.
$$

We shall show that

$$
g \in \mathcal{A} \text {. }
$$

Suppose on the contrary that $g \notin \mathcal{A}$. Then, according to Lemma 2, we can show that the function $g_{1}: I \rightarrow I$ defined by:

$$
g_{1}(x)=\left\{\begin{array}{lll}
\varphi(a) & \text { if } & x \leq a \\
g(x) & \text { if } & x \in(a, b) \\
\varphi(b) & \text { if } & x \geq b
\end{array}\right.
$$

does not belong to $\mathcal{A}$.

From Lemma 1 it follows that $g^{*}=g_{1 \|\left[\varphi\left(x_{0}\right)-\frac{\varepsilon}{4}, \varphi\left(x_{0}\right)+\frac{\varepsilon}{4}\right]}$ is not almost continuous, and so, there exists

a minimal
blocking

set

$K_{g} \cdot \subset I \times\left[\varphi\left(x_{0}\right)-\frac{\varepsilon}{4}, \varphi\left(x_{0}\right)+\frac{\varepsilon}{4}\right]$ of $g^{*}$. By Lemma 2 we deduce that the assumption of Lemma 3 are fulfils and so $(a, b) \cap K_{g^{*}}$ contains some nondegenerate interval $[c, d]$. Thus the set

$$
\begin{aligned}
& M=\left\{\left(x, \min \left\{y \in I:(c, d) \in K_{g^{*}}\right\}\right): x \leq c\right\} \cup \\
& \cup\left\{\left(x, \min \left\{y \in I:(d, y) \in K_{g^{*}}\right\}\right): x \geq d\right\} \cup \\
& \cup\left(K_{g^{*}} \cap\{(x, y): c \leq x \leq d\}\right)_{\min }
\end{aligned}
$$

is the graph of some function $\eta \in \mathbf{B}_{1}$. Let $B_{\eta} \in \mathcal{B}$ such that $\xi\left(B_{\eta}\right)=\eta$ and $z \in B_{\eta} \cap[c, d]$. Then $g^{*}(z)=g(z)=\eta(z)$ and, consequently, $\left(z, g^{*}(z)\right) \in K_{g^{*}}$, which contradicts the assumption that $K_{g^{*}}$ is the blocking set of $g^{*}$; thus condition (4) is satisfied.

We infer that:

$$
\varrho^{*}(\varphi, g) \leq \frac{\varepsilon}{2}
$$

However, by condition (5), we have

$$
K\left(g, \frac{\varepsilon}{2}\right) \subset K(\varphi, \varepsilon) .
$$

Now, we shall show that, for each function $\zeta \in \mathcal{A}_{1}$, we have $\zeta \notin K\left(g, \frac{e}{5}\right)$. Assume to the contrary that there exists $\zeta$ such that $\zeta \in K\left(g, \frac{\varepsilon}{5}\right) \cap \mathcal{A}_{1}$. Therefore, in the interval $(a, b)$ there lies some continiuty point $y_{0}$ of $\zeta$. We deduce that $\zeta\left(y_{0}\right) \in\left[\varphi\left(x_{0}\right)-\frac{\varepsilon}{4}, \varphi\left(x_{0}\right)+\frac{\varepsilon}{4}\right]$. Without loss of generality assume, that $\zeta\left(y_{0}\right) \in\left[\varphi\left(x_{0}\right), \varphi\left(x_{0}\right)+\frac{\varepsilon}{4}\right]$ (if $\zeta\left(y_{0}\right) \in\left[\varphi\left(x_{0}\right)-\frac{\varepsilon}{4}, \varphi\left(x_{0}\right)\right]$, then the proof is analogous). Thus there exists a nondegenerate interval $(p, q) \subset(a, b)$ such that $\zeta((p, q)) \subset\left(\varphi\left(x_{0}\right)-\frac{\varepsilon}{20}, 1\right]$. Let $B^{\prime} \in \mathcal{B}$ be a set such that the function $\xi\left(B^{\prime}\right)$ is constant and 
cqual to $\varphi\left(x_{0}\right)-\frac{f}{i}$ : moreover. let $s \in B^{\prime} \cap(p . q)$. Then $g(s)=\varphi\left(x_{0}\right)-\frac{\varepsilon}{1}\left(\xi(s)>\varphi\left(x_{0}\right)-\frac{\varepsilon}{20}\right)$. so $\varrho^{*}(g, \zeta) \geq \frac{5}{5}$, Which contradicts our assumption that $\zeta \in K\left(g, \frac{\varepsilon}{5}\right)$.

According to (3) and (6) and the above, we infer that

$$
i_{i}\left(t, R_{n}, . M \cup \mathcal{A}_{1}\right) \geq \frac{\psi_{n}}{10} .
$$

Applying (2). note that $\frac{\partial\left(t, R_{n}, 1 / \cup A_{1}\right)}{R_{n}} \geq \frac{2\left(t, R_{n}, 1\right)-\frac{1}{2^{n}} R_{n}}{10 R_{n}}$ (for $n$ sullicionly large). Thus, to conclude the prool. it sufficies to oluerve that:

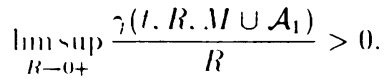

TSupported by KBN research grant PB $691 / 2 / 91$.

\section{References}

[1] Brown J.B.. Almost continuous Darboux functions and Reed's pointwise convergence criteria, Fund. Math. 86 (197.t) pp.219-26:3.

[2] Bruckner A.M., Differr ntiation of real functions, Springer- -Verlag (1978).

[3] Kellum K.R. . Almost contunuous functions on $I^{n}$, Fund. Math. 79 (1973), pp.213-215.

[4] Kellum K.R., Sums and lumuts of almost continuous functions, Coll. Math.31 (1974), pp.125128.

[5] Lipiński J.S., On a problem concerning the almost continuity, Zesz. Nauk. U.G. 4 (1979),pp.61-64.

[6] Natkaniec T., Almost continuity,Bydgoszcz (1992), pp.1-132.

[7] Reed C.S., Pointwise limits of sequences of functions, Fund. Math. 67 (1970), pp.183-193.

[8] Stallings J., Fixed point theorems for connectivity maps, Fund. Math. 47 (1959), pp.249-263.

[9] Stronska E., Algebraic structures generated by $T_{d}$ - quasi-continuous and almost continuous functions on $R^{m}$, Real Anal. Exch. 16 (1990-91), pp.169-176.

[10] Zajiček L., Sets of $\sigma$-porosity and sets of $\sigma$-porosity(q), Časopis Pěst. Mat. 101 (1976) pp.350-359.

[11] Zajiček L., Porosity, I-density topology and abstract density topologies, Real. Anal. Exch. 1 (1986-87), pp.313-326. 


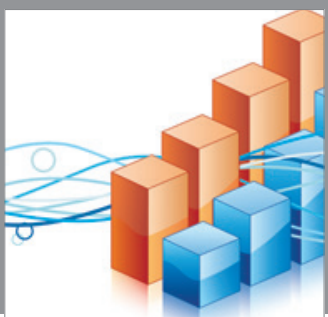

Advances in

Operations Research

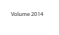

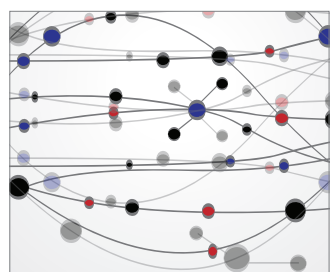

\section{The Scientific} World Journal
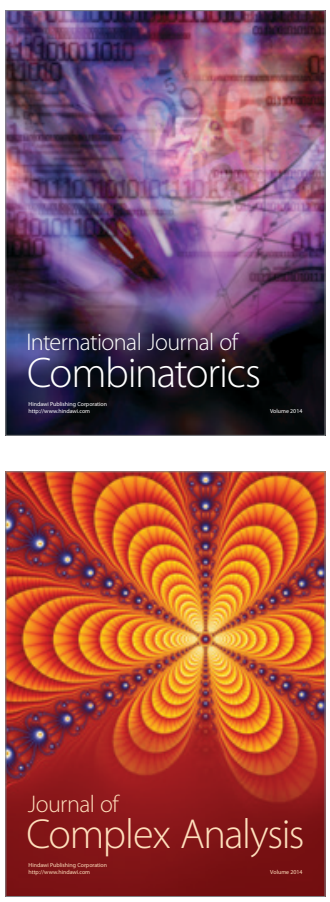

International Journal of

Mathematics and

Mathematical

Sciences
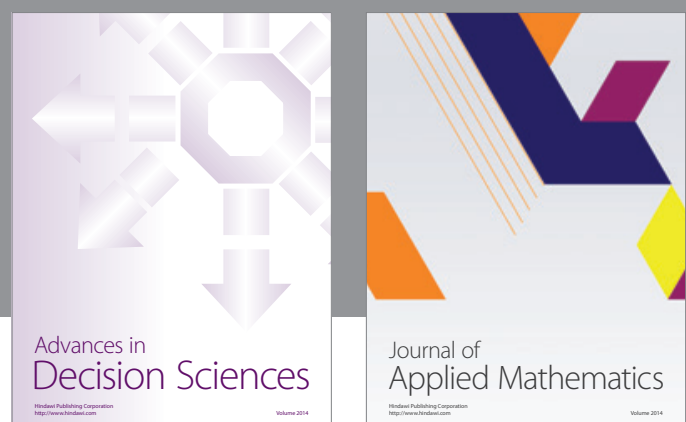

Journal of

Applied Mathematics
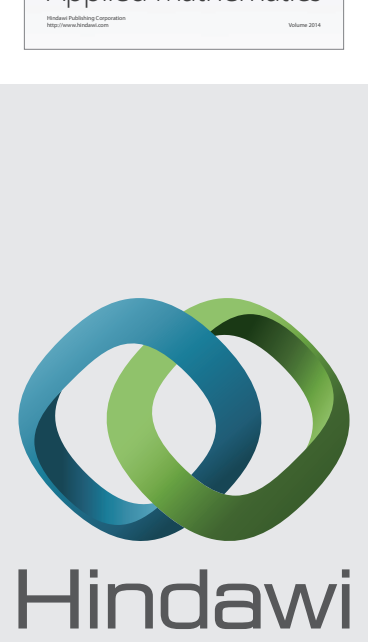

Submit your manuscripts at http://www.hindawi.com
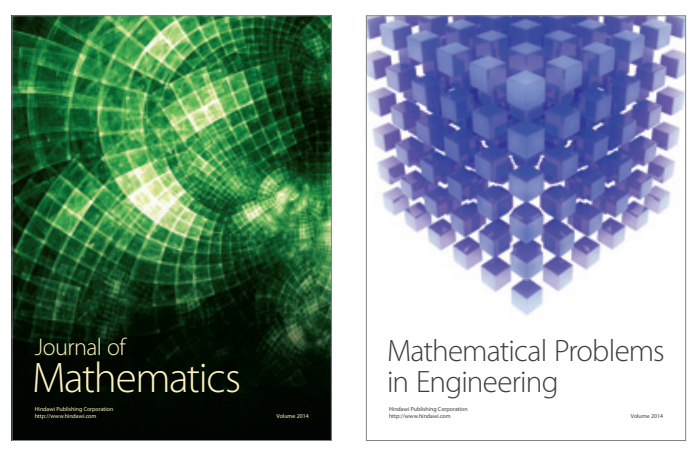

Mathematical Problems in Engineering
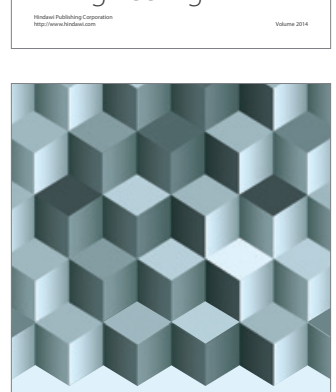

Journal of

Function Spaces
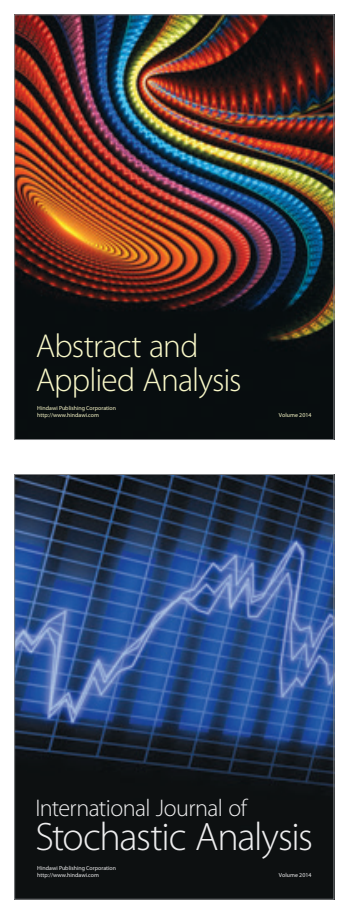

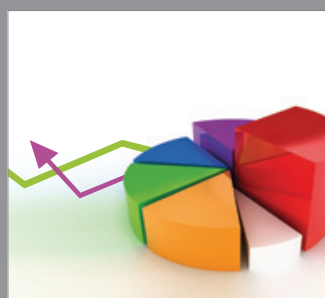

ournal of

Probability and Statistics

Promensencen
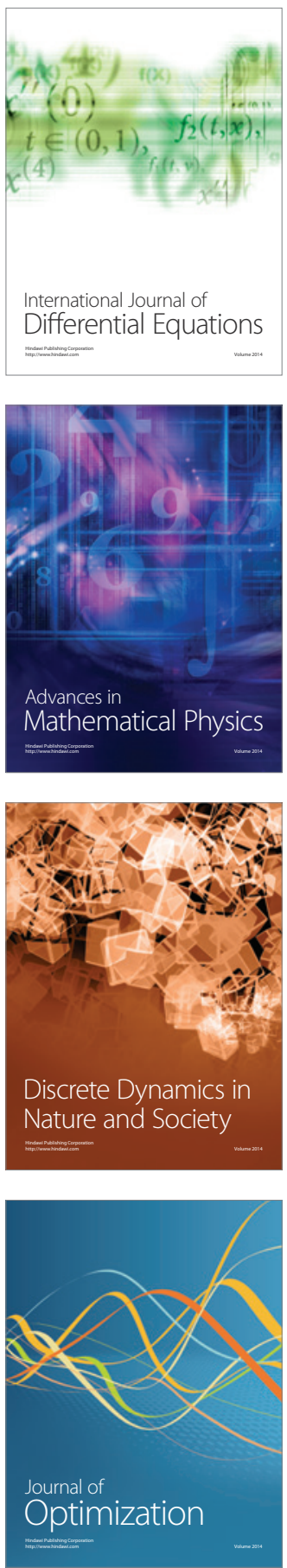